\title{
Wide spread of OXA-48-producing Enterobacteriaceae in Algerian hospitals: A four years' study
}

\author{
Nadjat Aggoune 1,3 , Hassiba Tali-Maamar ${ }^{2,3}$, Farida Assaous ${ }^{2}$, Badia Guettou ${ }^{2}$, Rym Laliam², Nabila \\ Benamrouche $^{2,3}$, Ali Zerouki ${ }^{1,3}$, Malek Naim ${ }^{1,3}$, Kheira Rahal ${ }^{2,3}$ \\ ${ }^{1}$ Laboratoire de Microbiologie, Hôpital central de l'armée Mohammed Seghir Nekkache, Alger, Algérie \\ ${ }^{2}$ Laboratoire de Bactériologie médicale et de surveillance de la résistance aux antibiotiques, Institut Pasteur \\ d'Algérie Alger, Algérie \\ ${ }^{3}$ Université d'Alger, Faculté de médecine, laboratoire de microbiologie, Alger, Algérie
}

\begin{abstract}
Introduction: The aim of this study was to investigate the presence of carbapenemase-producing Enterobacteriaceae (CPE) in Algerian hospitals and to characterize the molecular types of carbapenemases found.

Methodology: During a four years study lasting between 2012 and 2015, 81 strains of Enterobacteriaceae with reduced susceptibility to carbapenems were collected from different hospitals. Carbapenemase genes were detected by PCR. Multi locus sequence typing was used to study genetic relationships between carbapenemase- producing Klebsiella pneumoniae isolates.

Results: Among 56 confirmed CPE, bla oxA-48 was detected in $98.21 \%$ of isolates. Two isolates co-expressed NDM, and a single one was only an NDM producer. The strains displayed various susceptibility patterns to antibiotics with variable levels of resistance to carbapenems. Multilocus sequence typing (MLST) revealed the presence of multiple sequence types in circulation.

Conclusions: This report highlights the wide distribution of several clones of OXA-48-producing Enterobacteriaceae in Algeria. Urgent action should be taken to avoid epidemic situations.
\end{abstract}

Key words: Carbapenemase; OXA-48; NDM; Klebsiella pneumoniae; sequence type; Algeria.

J Infect Dev Ctries 2018; 12(11):1039-1044. doi:10.3855/jidc.9692

(Received 15 August 2017 - Accepted 10 January 2018)

Copyright (C) 2018 Aggoune et al. This is an open-access article distributed under the Creative Commons Attribution License, which permits unrestricted use, distribution, and reproduction in any medium, provided the original work is properly cited.

\section{Introduction}

Over the last decade, carbapenem resistance among Enterobacteriaceae has been extensively observed worldwide, seriously compromising the usage of carbapenems. Carbapenems are considered the drugs of choice in treating severe infections caused by multidrug resistant strains, especially those expressing extended-spectrum-beta-lactamases.

Carbapenemase production represents the most important mechanism that affects carbapenem activity. Among the Enterobacteriaceae, three molecular types of carbapenemases are of major clinical importance since they are frequently associated with severe nosocomial infections. These are KPC (Ambler class A), NDM (class B) and OXA-48 (class D). These enzymes are widely distributed all over the world. However, specific reservoirs are identified for each type. KPC is mainly found in USA, China, Greece, and Italy. NDM is mainly present in India and Pakistan, while OXA-48 seems to be endemic in Turkey and in a lot of countries of the Mediterranean area. It is also largely found in North Africa [1].

In Algeria, data regarding carbapenemaseproducing Enterobacteriaceae (CPE) are scarce since only five isolates producing VIM-19 carbapenemase and a single strain expressing bla oxA-48 were recovered prior to 2012 [2,3].

Here we describe the wide spread of carbapenemase-producing Enterobacteriaceae (CPE) in our country during the period between January 2012 and December 2015.

\section{Methodology}

Bacterial isolates

During the reporting period, we conducted a multicenter study that aimed to investigate the presence of CPE and to characterize carbapenemase genes present in Algerian hospitals. Twelve hospital laboratories collaborated in the study. The participants were consequently invited to send their Enterobacteriaceae isolates that showed reduced 
susceptibility to ertapenem (intermediate and resistant strains according to Clinical and Laboratory Standards Institute-CLSI- breakpoints) [4].

All the isolates were gathered in the central laboratory (Laboratoire de bactériologie médicale et de surveillance de la résistance aux antibiotiques; Institut Pateur of Algeria), where their identification and antimicrobial resistance profiles were verified. The isolates were then submitted to different phenotypic and molecular testing.

\section{Bacterial identification and antimicrobial susceptibility testing}

Isolates were identified by using API 20E system (bioMérieux, Marcy l'Etoile, France) and antimicrobial susceptibility testing was performed by the disk diffusion method according to CLSI guidelines for most of the antibiotics [4]. Minimum Inhibitory Concentrations (MICs) for carbapenems (ertapenem, meropenem and imipenem) and tigecycline were determined by using E test strips (bioMérieux, Marcy l'Etoile, France). The interpretation of results was made according to CLSI breakpoints (for most antibiotics) and EUCAST breakpoints for tigecycline. [5]

\section{Phenotypic detection of carbapenemases and extended spectrum beta-lactamases (ESBLs)}

Phenotypic study of different carbapenemases included a modified Hodge test (MHT), EDTA and temocillin assays $[4,6-7]$. ESBL production was studied using the double disk method by testing both cefotaxime ceftazidime, aztreonam and cefepime alone and in combination with clavulanic acid. A $>5 \mathrm{~mm}$ increase in a zone diameter for either antimicrobial agent tested in combination with clavulanic acid versus its zone when tested alone confirms an ESBLproducing strain.

\section{Molecular analysis of carbapenemase genes}

The main carbapenemase encoding genes (bla IMP, bla vIM, bla OXA-48, bla NDM and bla KPC) were investigated by polymerase chain reaction (PCR) using previously designed primers [8], followed by sequencing analysis (Sanger's technique) in order to determine the exact variant of the genes. Briefly, amplicons were purified using the PureLink Quick PCR purification kit (Invitrogen, Applied Biosystems, Vilnius, Lithuania) according to the manufacturer's instructions and sequenced using the BigDye Terminator ready reaction mix v3.1 (Perkin-Elmer Applied Biosystems, Austin, TX, USA), following the manufacturer's instructions. The forward and reverse sequences were aligned using the CLC WorkBench7.0 software. The type of each identified gene was obtained by submitting aligned DNA sequences in the BLAST (https://blast.ncbi.nlm.nih.gov/Blast.cgi).

Typing

The genetic relationship between the 15 different $K$. pneumoniae isolates was determined by MLST using internal fragments of seven housekeeping genes ( $g a p A$, inf $B, m d h$, pgi, phoE, rpoB and tonB) according to the Diancourt et al. method (amplification and sequencing) [9]. The generated DNA sequences were deposited in the MLST database (http://bigsdb.web.pasteur.fr/klebsiella/klebsiella.html) to obtain allelic profiles and sequence type (ST) designations.

The minimum spaning tree of the 15 analyzed strains was built using Bionumerics version 7.5 software. In order to assess a clonal analysis, a single isolate for each ST identified in this work, as well as STs available in the MLST database at the time of the study, were included using the eBurst version 3 program (www.mlst.net). A clonal complex or eBurst group was defined as a cluster of isolates sharing six of seven alleles.

\section{Results}

Bacterial isolates

Of the 81 isolates received during the study period, $56(69,13 \%)$ were carbapenemase producers. CPE were recovered from 54 patients hospitalized in 11 different hospitals located in the cities of Algiers, Blida (west of Algiers), Tizi Ouzou (east of Algiers), Oran (west Algeria) and Constantine (east Algeria). Strains were associated with different clinical infections including urinary tract infections $(\mathrm{n}=17)$, wound infections $(n=13)$, pneumonia $(n=11)$, bacteremia $(n$ $=8)$, catheter sepsis $(\mathrm{n}=3)$, intra-abdominal infections $(n=2)$, vaginal infections $(n=2)$. Three other strains were found in fecal samples belonging to asymptomatic patients. Among the carbapenemase producing isolates, $K$. pneumoniae represented the most prevalent species, followed by $E$. coli and $E$. cloacae with $83,93 \%$ (n = $47), 8,93 \% \%(\mathrm{n}=5)$ and $7,14 \%(\mathrm{n}=4)$ of cases respectively.

\section{Antimicrobial susceptibility}

All carbapenemase- producing isolates were multiresistant but displayed various antimicrobial susceptibility patterns. Seventy-three percent $(n=41)$ of the tested isolates were resistant to cefotaxime, $62.5 \%(\mathrm{n}=35)$ to ceftazidime and $60.71 \%$ to aztreonam 
Table 1. Antimicrobial susceptibility patterns of CPE isolates.

\begin{tabular}{ccc}
\hline Antibiotics & Total number of isolates & Resistant N (\%) \\
\hline Cefotaxime & 56 & $41(73)$ \\
Ceftazidime & 56 & $35(62.5)$ \\
Aztreonam & 56 & $34(60.71)$ \\
Ertapenem & 56 & $55(98.21)$ \\
Meropenem & 56 & $26(46.43)$ \\
Imipenem & 56 & $21(37.5)$ \\
Gentamicin & 56 & $36(64.28)$ \\
Amikacin & 56 & $13(23.21)$ \\
Tobramycin & 56 & $34(60.71)$ \\
Netilmicin & 56 & $17(30.35)$ \\
Nalidixic acid & 56 & $29(51.78)$ \\
Ciprofloxacin & 56 & $26(46.43)$ \\
Levofloxacin & 56 & $13(23.21)$ \\
Cotrimoxazole & 56 & $38(67.85)$ \\
Chloramphenicol & 56 & $13(23.21)$ \\
Tigecycline & 56 & $3(5.36)$ \\
\hline
\end{tabular}

$(\mathrm{n}=34)$ (Table 1). Regarding the carbapenems, 62.5\% $(\mathrm{n}=35)$ of the strains remained susceptible to imipenem (MIC range $0.25-1 \mathrm{mg} / \mathrm{L})$ and $53.57 \%(\mathrm{n}=30)$ to meropenem (MIC range 0.19-1 $\mathrm{mg} / \mathrm{L}$ ). On the other hand, resistance to ertapenem was observed in $98.21 \%$ $(\mathrm{n}=55)$ of cases with MIC range from 1.5 to $32 \mathrm{mg} / \mathrm{L}$ and MIC $90=32 \mathrm{mg} / \mathrm{L}$ (Table 1 and 2). Among aminoglycosides, amikacin and netilmicin showed the highest activities with $76.78 \%(\mathrm{n}=43)$ and $69.64 \%(\mathrm{n}$ $=39$ ) of sensitive strains respectively, while gentamicin and tobramycin showed lower percentages of sensitivity: $35.71 \%(\mathrm{n}=20)$ and $39.28 \%(\mathrm{n}=22)$ respectively. Levofloxacin was the most active quinolone with a sensitivity rate of $76.78 \%(n=43)$ while $53.57 \%(\mathrm{n}=30)$ of isolates were sensitive to ciprofloxacin and $48.41 \%(\mathrm{n}=27)$ to nalidixic acid. Thirty-eight $(67.85 \%)$ isolates were resistant to cotrimoxazole, while chloramphenicol remained active against $76.78 \%(\mathrm{n}=43)$ of the tested strains. Tigecycline was the most active drug with $94.64 \%$ ( $n=$ 53) of sensitive isolates, MIC range from 0.125 to 4 $\mathrm{mg} / \mathrm{L}$ and $\mathrm{MIC} 90=2 \mathrm{mg} / \mathrm{L}$ ) (Table 1 and 2). Coexpression of ESBL was noticed in $33(58.92 \%)$ isolates.

\section{Molecular detection}

The molecular identification showed that OXA-48 is by far the most frequent carbapenemase among the
Enterobacteriaceae isolates in Algeria $(98,21 \%, \mathrm{~N}=$ 55). Also, NDM were found in 3 isolates including two co-expressing bla OXA-48. Sequencing identified the presence of the allele OXA-48 for 4 strains and the variant NDM-1 for two strains.

\section{Molecular epidemiology}

Multilocus sequence typing analysis allowed us to identify 11 different sequence types (STs) among the 15 analyzed $K$. pneumoniae isolates. Sequence type ST147 $(\mathrm{n}=3)$ was mainly found in the hospital central de l'armée of Algiers and the university hospital of Constantine. It was linked twice to the co-production of NDM and OXA-48 carbapenemase. Four different other STs; ST101 $(n=2)$, ST14 $(n=2), \operatorname{ST} 336(n=1)$ and ST1412 $(n=1)$ were also identified in different wards of the hospital central de l'armée of Algiers (intensive care, surgery and burns). Sequence types ST13 (n=1), ST485 (n=1), ST870 $(\mathrm{n}=1)$ and ST1593 $(\mathrm{n}=1)$ were linked to strains isolated from 4 different hospitals of Algiers region, while ST348 $(\mathrm{n}=1)$ was detected in university hospital of Blida and ST405 ( $\mathrm{n}=$ 1) in the military hospital of Oran.

In summary, different STs were identified and were not linked to a geographical origin (hospital or area) (Figure 1).

Clonal analysis classified isolates into two clonal complexes designated by eBurst groups. Ten strains

Table 2. MICs for ertapenem, meropenem, imipenem and tigecycline of CPE isolates.

\begin{tabular}{ccccc}
\hline & Ertapenem & Meropenem & Imipenem & Tigecycline \\
\hline MIC range $(\mu \mathrm{g} / \mathrm{mL})$ & $1.5-32$ & $0.19-32$ & $0.25-32$ & $0,125-4$ \\
MIC50 $(\mu \mathrm{g} / \mathrm{mL})$ & 32 & 3 & 1.5 & 1 \\
MIC90 $(\mu \mathrm{g} / \mathrm{mL})$ & 32 & 32 & 32 & 2 \\
\hline
\end{tabular}


assigned to different STs clustered in the eBurst group I, while the three isolates belonging to ST147 were part of eBurst group III. Two strains belonged to ST 1593 and ST348 respectively were singleton (Table 3).

\section{Discussion}

Since the inclusion of ertapenem in the susceptibility testing in our country from 2012, we are facing more and more clinical situations of isolating strains with decreased susceptibility to carbapenems in Enterobacteriaceae, as noticed throughout the world. In Algeria, the prevalence of resistance to third generation cephalosporins among nosocomial $K$. pneumoniae isolates reached $58.93 \%$ in 2015 while carbapenem resistance was around $2.33 \%$ (data of the Algerien Antimicrobial Resitant

Network http://www.sante.dz/aarn/) . This latter digit is likely to increase in the future in view of the strong pressure exerted by carbapenems for the treatment of serious infections due to cephalosporin-resistant enterobacteriaceae.

The data presented here, based on a 4-years survey, provide the first insight into the widespread occurrence of CPE in Algeria where carbapenemase production seems to be the main mechanism causing carbapenem resistance.

It is important to remember that carbapenem resistance in enterobacteriaceae can also be due to the combination of impermeability and production of extended-spectrum beta-lactamase (ESBL) or an overexpressed AmpC enzyme.

The isolates found in the study were associated with a large range of nosocomial infections of variable gravity. K. pneumoniae was by far the most important species.
Figure.1. Minimum spanning tree of the MLST of the 15 carbapenemase producing Klebsiella pneumoniae isolates.

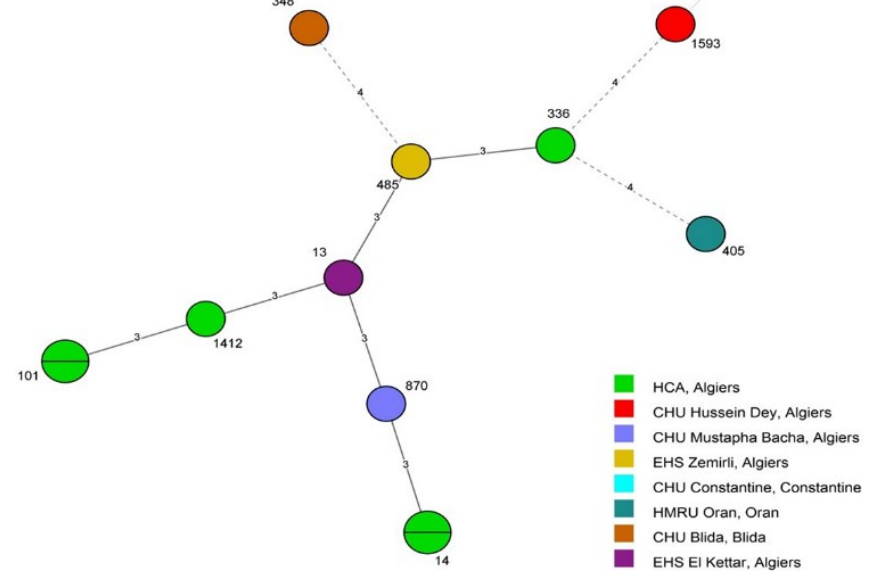

Each circle corresponds to an ST. The area of each circle corresponds to the number of isolates. Each ST is color coded according to its corresponding geographical origin. The relationships between strains are indicated by the connections between the isolates, and the numbers between the circles indicate the number of allelic differences. The black lines connecting the ST pairs indicate that they differ by three alleles (thick lines), four alleles (dotted lines), and five alleles (thin lines).

Carbapenems are still used for the treatment of CPE infections in Algeria because of the lack of other therapeutic options (piperacillin- tazobactam, tigecycline and intravenous fosfomycin are unavailable). Some CPE strains coproducing ESBL found in this work exhibited high levels of resistance to carbapenems. This is consequently of great clinical concern. Colistin remains the last drug for the treatment of infections caused by CPE in Algeria. Thus, resistance to this antibiotic should absolutely be monitored.

Table 3. Sequence type and eBurst group of the $15 \mathrm{CPE}$ isolates.

\begin{tabular}{cccccc}
\hline $\begin{array}{c}\text { eBurst } \\
\text { group }\end{array}$ & $\begin{array}{c}\text { Sequenc } \\
\text { e type }\end{array}$ & Allelic profile & $\begin{array}{c}\text { Number of } \\
\text { isolates }\end{array}$ & Carbapenemase type & Geographical origin \\
\hline 1 & 13 & $2-3-1-1-10-1-19$ & 1 & OXA-48 & EHS El Kettar, Algiers \\
1 & 485 & $2-1-1-1-7-1-12$ & 1 & OXA-48 & OHS Zemirli, Algiers \\
1 & 336 & $2-1-1-1-72-4-4$ & 1 & OXA-48 & HCA, Algiers \\
1 & 1412 & $2-5-1-1-4-1-18$ & 1 & OXA-48 Algiers \\
1 & 14 & $1-6-1-1-1-1-1$ & 2 & OXA-48 & HCA, Algiers \\
1 & 405 & $2-1-62-3-10-4-110$ & 1 & OXA-48 & HMRUO, Oran \\
1 & 870 & $2-6-1-1-147-1-31$ & 1 & OXA-48 & Mustapha Bacha, Algiers \\
1 & 101 & $2-6-1-5-4-1-6$ & 2 & OXA-48+NDMOXA- Algiers \\
3 & 147 & $3-4-6-1-7-4-38$ & 3 & $48+$ NDMOXA-48 & HCA, AlgiersCHU Constantine, \\
& 1593 & $4-1-11-1-9-4-59$ & 1 & OXA-48 & ConstantineHCA, Algiers \\
& 348 & $2-1-20-1-12-15-16$ & 1 & OXA-48 & CHusein Dey, Algiers \\
\hline
\end{tabular}

EHS: Etablissement Hospitalier Universitaire; HCA: Hôpital Central de l'Armée; CHU: Centre Hospitalo Universitaire; HMRU: Hôpital Militaire Régional Universitaire. 
Our results showed a very large prevalence of OXA-48 type carbapenemase that could be considered endemic, and a rare presence of the NDM type amongst carbapenem non sensitive Enterobacteriaceae strains. This report consequently confirms the recent observations in neighboring countries [10-13]. Regarding NDM producers ( 3 isolates), in the absence of travel history data of the patients and as it is not common for Algerian patients to travel to the Indian subcontinent, we cannot dismiss the presence of autochthonous cases as previously described elsewhere [14].

Muti locus sequence typing among $K$. pneumoniae isolates revealed the presence of the international and multi resistant clones ST147, ST101, and ST14. ST101 is a successful clone of OXA-48 type carbapenemase and it has already been found in several countries of the Mediterranean area including those surrounding Algeria (Morocco, Libya and Tunisia) [13-15]. ST147 is a clone frequently associated to the production of different types of carbapenemases around the world (KPC, VIM, NDM and OXA-48) [15,16]. ST14 is a clone found in OXA-48 producers in Turkey [15-17] and NDM producers in India and United Kingdom [18]. Two isolates belonged to ST307 and ST405. Those STs were also found in Morocco (OXA-48) $[15,19]$ and Spain (OXA-48, OXA-244, OXA-245) $[15,20]$. The other clones found in this study were rarely associated to carbapenemase production. The current spread of $b_{\text {OXX-48 }}$ in K. pneumoniae is not simply linked to its diffusion among successful clones, but it is mainly due to its presence on a highly-transmissible, selfconjugative IncL/M plasmid [21].

The majority of the identified STs clustered into two major clonal complexes: I ( 10 strains belonging to different STs) and III (3 strains belonging to ST147).

\section{Conclusion}

This report has documented the widespread of OXA-48 producing Enterobacteriaceae in our hospitals and the genetic diversity of circulating clones. However, the major limitation is the absence of detailed clinical and epidemiological data that can allow us to appreciate the real impact of CPE on infected patients, outcomes and efficiency of administered treatment. Also, there is an urgent need to identify CPE to ensure adapted antibiotherapy and to implement control measures to avoid further dissemination.

\section{Acknowledgements}

The authors would like to thank the following microbiologists for sending strains and for technical support: Djamel Tiouit, Fatma Zohra Henniche, Doria Bensersa, Amine Abdouni, Imène Balout, Abdeslam Ladouari, Kahina Kacimi, Amira Ryma Boutella, Célia Kasmi, Randa Gouigah (Hôpital Central de l'Armée, Algiers, Algeria); Mounira Nabila Ouar Korichi (Institut Pasteur d'Algérie, Algiers, Algeria); Kamel Kezzal (Institut Pasteur d'Algérie, Algiers, Algeria); Nadjia Ramdani- Bouguessa, Mohamed Tazir, Fazia Djennane, Mohamed Bachtarzi (Centre Hospitalier Universitaire Mustapha Bacha, Algiers, Algeria); Rachid Bellouni, Samira Berouaken, Siham Azrou (Centre Hospitalier Universitaire Frantz Fanon,Blida, Algeria); Moufida Hamidi (Etablissement Hospitalier Specialisé Salim Zemirli, Algiers, Algeria) ; Kaddour Benlabed, Chafia Bentchouala (Centre Hospitalier Universitaire Ben Badis, Constantine, Algeria) ; Salim Khemissi (Hôpital Militaire Régional Universitaire de Constantine, Algeria) ; Ahmed Amine Louail, Lahcène Benmahdi (Hôpital Militaire Régional Universitaire d'Oran, Algeria) ; Zehor Guechi, Sadjia Mahrane (Centre Hospitalier Universitaire Nafissa Hamoud, Algiers, Algeria); Amina Azzam (Centre Hospitalier Universitaire Nedir Mohamed, Tizi Ouzou, Algeria); Wahiba Amhis (Etablissement Hospitalier Specialisé Bologhine Ibn Ziri, Algiers, Algeria); Houria Ammari (Centre Hospitalier Universitaire Isaad Hassani, Algiers,Algeria).

\section{References}

1. Nordmann P, Poirel L (2014) The difficult to control spread of carbapenemase producers among Enterobacteriaceae worldwide. Clin Microbiol Infect 20: 821-830.

2. Robin F, Aggoune-Khinache N, Delmas J, Naim M, Bonnet R (2010) Novel VIM Metallo- $\beta$-lactamase variant from clinical isolates of Enterobacteriaceae from Algeria. Antimicrob Agents Chemother 54: 466-470.

3. Aggoune N, Tali -Maamar H, Assaous F, Benamrouche N, Naim M, Rahal K (2014) Emergence of plasmid mediated carbapenemase OXA-48 in a Klebsiella pneumoniae strain in Algeria. Jgar 2: 327-329.

4. Clinical and Laboratory Standards Institute (CLSI) (2016). Performance standards for antimicrobial susceptibility testing; 26th informational supplement CLSI document M-100- S 26 (ISBN 1-56238-923-8).

5. European Committee on Antimicrobial Susceptibility Testing (EUCAST) Clinical breakpoints: Breakpoint tables for bacteria. Available: http://www.eucast.org/clinical_breakpoints/. Accessed 2 March 2015.

6. Picão RC, Andrade SS, Nicoletti AG, Campana EH, Moraes GC, Mendes RE, Gales AC (2008) Metallo-beta-lactamase detection: comparative evaluation of double-disk synergy versus combined disk tests for IMP-, GIM-, SIM-, SPM-, or VIM-producing isolates. J Clin Microbiol 46: 2028-2037.

7. Huang T, Poirel L, Bogearts P, Berhin C, Nordmann P, Glupczynski Y (2014) Temocillin and piperacillin/tazobactam resistance by disk diffusion as antimicrobial surrogate markers for the detection of carbapenemase-producing 
Enterobacteriaceae in geographical areas with high prevalence of OXA-48 producers. J Antimicrob Chemother 69: 445-450.

8. Poirel L, Walsh TR, Curvillier, Nordmann P (2014) Multiplex PCR for detection of acquired carbapenemase genes. Diagn Microbiol Infect Dis 70: 119-123.

9. Diancourt L, Passet V, Verhoef J, Grimont P, Brisse S (2005) Multilocus sequence typing of Klebsiella pneumoniae nosocomial isolates. J Clin Microbiol 43: 4178-4182.

10. Barguigua A, El Otmani F, Lakbakbi El Yaagoubi F, Talmi M, Zerouali K, Timinouni M (2013) First report of a Klebsiella pneumoniae strain coproducing NDM-1, VIM-1 and OXA-48 carbapenemases isolated in Morocco. APMIS. 121: 675-677.

11. Ktari S, Mnif B, Louati F, Rekik S, Mezghani S, Mahjoubi F, Hammami A (2011) Spread of Klebsiella pneumoniae isolates producing OXA-48 $\beta$ - lactamase in a Tunisian university hospital. J Antimicrob Chemother 66: 1644-1646.

12. Ben Nasr A, Decré D, Compain F, Genel N, Barguellil F, Arlet G (2013) Emergence of NDM-1 in association with OXA-48 in Klebsiella pneumoniae from Tunisia. Antimicrob Agents Chemother 57: 4089-4090.

13. Lafeuille E, Decré D, Mahjoub-Messai F, Bidet P, Arlet G, Bingen E (2013) OXA-48 carbapenemase-producing Klebsiella pneumoniae isolated from Libyan patients. Microb Drug Resist 19: 491-497.

14. Nordmann P, Couard JP, Sansot D, Poirel L (2012). Emergence of an autochthonous and community acquired NDM-1 producing Klebsiella pneumoniae in Europe. Clin Infect Dis 54: 150-151.

15. Potron A, Poirel L, Rondinaud E, Nordmann P (2013) Intercontinental spread of OXA-48 beta-lactamase producing Enterobacteriaceae over a 11-year period, 2001 to 2011. Euro Surveill 18: 20549.

16. Zowawi HM, Forde BM, Alfaresi M, Alzarouni A, Farahat Y, Chong TM, Yin WF, Chan KG, Li J, Schembri MA, Beatson SA, Paterson DL (2015) Stepwise evolution of pandrugresistance in Klebsiella pneumoniae. Sci Rep 5: 15082.
17. Cuzon G, Ouanich J, Gondret R, Naas T, Nordmann P (2011) Outbreak of OXA-48-positive carbapenem-resistant Klebsiella pneumoniae isolates in France. Antimicrob Agents Chemother 55: 2420-2423.

18. Giske CG, Fröding I, Mehedi Hasan C, Turlej-Rogacka A, Toleman M, Livermore D, Woodford N, Walsh TR (2012) Diverse sequence types of Klebsiella pneumoniae contribute to the dissemination of blaNDM-1 in India, Sweden, and the United Kingdom. Antimicrob Agents Chemother 56: 27352738.

19. Girlich D, Bouihat N, Poirel L, Benouda A, Nordmann P (2014) High rate of faecal carriage of extended-spectrum $\beta$ lactamase and OXA-48 carbapenemase-producing Enterobacteriaceae at a university hospital in Morocco. Clin Microbiol Infect 20: 350-354.

20. Oteo J, Hernandez JM, Espasa M, Fleites A, Saez D, Bautista $\mathrm{V}$, Pérez-Vázquez $\mathrm{M}$, Fernández-García MD, DelgadoIribarren A, Sánchez-Romero I, García-Picazo L, Miguel MD, Solís S, Aznar E, Trujillo G, Mediavilla C, Fontanals D, Rojo S, Vindel A, Campos J (2013) Emergence of OXA-48producing Klebsiella pneumoniae and the novel carbapenemases OXA-244 and OXA-245 in Spain. J Antimicrob Chemother 68: 317-321.

21. Poirel L, Bonnin RA, Nordmann P (2012) Genetic features of the widespread plasmid coding for the carbapenemase OXA48. Antimicrob Agents Chemother 56: 559-562.

\section{Corresponding author}

Dr Nadjat Aggoune

Laboratoire de Microbiologie

Hôpital central de l'armée, Mohamed Seghir Nekkache BP 244

Kouba, Algiers, Algeria.

Phone:(213) 661791220.

Email: nadjetaggoune@yahoo.fr

Conflict of interests: No conflict of interests is declared. 